\title{
Ambulante Verhaltenstherapie bei Trichotillomanie: Überblick, Störungsmodell und Fallbeispiel
}

\author{
A. Neudecker ${ }^{\mathrm{a}} \quad$ M. Rufer ${ }^{\mathrm{b}}$ \\ a Bezirkskrankenhaus Landshut \\ b Zentrum für Psychosoziale Medizin, Klinik und Poliklinik für Psychiatrie und Psychotherapie, Universitätsklinikum Hamburg-Eppendorf, \\ Deutschland
}

\section{Schlüsselwörter}

Trichotillomanie · Verhaltenstherapie

Impulskontrollstörung · Zwangsspektrumsstörung

\section{Zusammenfassung}

Trichotillomanie, laut diagnostischer Kategorisierung eine Störung der Impulskontrolle, ist eine in ihrer Häufigkeit bisher unterschätzte Erkrankung. Obwohl sie übereinstimmend zu den Störungen des Zwangsspektrums gezählt wird, besteht hinsichtlich Ätiologie, Prävalenz, Neurobiologie und effizienter Therapieverfahren noch keineswegs Klarheit. Besonders schwierig ist, dass die Trichotillomanie sowohl zwanghafte wie auch impulsive und damit positiv verstärkte Verhaltensanteile enthält. Verhaltenstherapie und Behandlung mit selektiven Serotonin-Wiederaufnahmehemmern gelten derzeit als Verfahren der Wahl. Nach einer Einführung und einer kurzen Darstellung des Forschungsstandes zur pharmakologischen Therapie wird ein kritischer Überblick über bisherige Studien zur verhaltenstherapeutischen Behandlung gegeben. Schwerpunkt ist die Diskrepanz zwischen Komplexität der Störung und meist ausschließlich symptomzentrierten Therapieansätzen. In einem ausführlichen Störungsmodell, das durch ein Fallbeispiel illustriert wird, wird nicht nur auf die Verhaltensebene, sondern auch auf Komorbidität, auslösende und aufrechterhaltende Bedingungen eingegangen. Die anhand dieses Modells durchgeführte multimodale Verhaltenstherapie wird beschrieben und diskutiert.

*Diese Publikation entstand auf der Basis der früheren Tätigkeit der Autorin an der Verhaltenstherapie-Ambulanz des Universitätsklinikums Hamburg-Eppendorf.

\begin{tabular}{ll}
\hline KARGER & @ 2004 S. Karger GmbH, Freiburg \\
Fax +49 76145207 14 & Accessible online at: \\
$\begin{array}{l}\text { E-mail Information@Karger.de } \\
\text { www.karger.com }\end{array}$ & www.karger.com/ver
\end{tabular}

Key Words

Trichotillomania - Behavior therapy - Impulse control disorder . Obsessive-compulsive spectrum disorder

\section{Summary}

Behavior Therapy of Trichotillomania: Review,

Behavioral Analysis, and Case History

Trichotillomania, which is categorized as impulse control disorder, is not as rare as has long been suspected. It is usually regarded as an obsessive-compulsive spectrum disorder, however, up to now findings about etiology, prevalence, neurobiology and efficient treatment methods have been inconsistent. Trichotillomania has both compulsive as well as impulsive features with mechanisms of positive reinforcement. Behavior therapy and treatment with selective serotonin reuptake inhibitors proved to be most helpful. After an introduction and a short review concerning the pharmacological treatment, a critical review of previous studies on behavior therapy in trichotillomania is given. The main focus lies on the discrepancy between the complex disorder on the one hand and the predominant use of isolated symptom-oriented techniques on the other hand. As an alternative approach, a multimodal behavior therapy is illustrated by a case history, comprising the behavioral analysis of and the interventions for the symptom behavior as well as for comorbidity, traumatic biographical events and maintaining variables. 


\section{Einleitung}

Trichotillomanie wird sowohl im ICD-10 als auch im DSM-IV als «Störung der Impulskontrolle» klassifiziert (F 63.3 bzw. 312.39). Hauptmerkmale sind der als unkontrollierbar erlebte Impuls, sich (meist einzelne) Haare vom Kopf oder anderen behaarten Körperstellen auszureißen, innere Anspannung vor und/oder Gefühl von Erleichterung nach der Handlung sowie eine durch das Haareausreißen bedingte signifikante Einschränkung der Lebensqualität. Entgegen anders lautender Vermutungen ist Trichotillomanie bei Erwachsenen mit einer Lebenszeitprävalenz von $1,6 \%$ bei Männern und 3,5\% bei Frauen [Christenson et al., 1991d] nicht selten. Im klinischen Setting überwiegt der Anteil an Frauen deutlich, weshalb in diesem Artikel durchgehend die weibliche Form «Patientinnen» verwandt wird. Hypothesen hierzu beziehen sich überwiegend auf geschlechtsspezifische Unterschiede im Hilfesuchverhalten [Christenson et al., 1994]. Es besteht eine hohe Komorbidität mit affektiven, Angst-, Abhängigkeits- und Essstörungen [Christenson, 1995] sowie zu Persönlichkeitsstörungen [Christenson et al., 1992; Schlosser et al., 1994; Swedo und Leonard, 1992], aber keine ist spezifisch für Trichotillomanie. Phänomenologisch wird Trichotillomanie als «Störung des Zwangsspektrums» [Hollander und Wong, 1995] betrachtet, wobei eine Reihe von deutlichen Unterschieden zur reinen Zwangsstörung bestehen [Christenson et al., 1991a; Stanley et al., 1992]. Besonders hervorzuheben ist, dass der Genuss als kurzfristig positive Konsequenz beim Haareausreißen eine wichtige Rolle spielt. Er entsteht entweder direkt beim Ausreißen eines Haares oder, was häufiger der Fall ist, während der folgenden oralen Beschäftigung mit der Haarwurzel. Vielen Patientinnen fällt es äußerst schwer, darauf zu verzichten, was erheblichen Einfluss auf die Therapiemotivation hat.

Als Therapiemethoden der Wahl werden Verhaltenstherapie und Pharmakotherapie betrachtet. Im Folgenden wird kurz der aktuelle Forschungsstand bezüglich der pharmakologischen Behandlungsoptionen zusammengefasst und anschlieBend ausführlich die Anwendung von Verhaltenstherapie bei Trichotillomanie dargestellt.

\section{Exkurs: Pharmakotherapie bei Trichotillomanie}

Die diagnostische Zuordnung der Trichotillomanie zu den Störungen des Zwangsspektrums spricht für eine Behandlung mit selektiven SerotoninWiederaufnahmehemmern (SSRI) oder Clomipramin (SRI), da mehrere dieser Störungen bevorzugt auf serotonerge Antidepressiva ansprechen. Die Ergebnisse von Therapiestudien mit (S)SRI zur Trichotillomanie sind allerdings gemischt. In 3 kontrollierten Untersuchungen mit Clomipramin [Ninan et al., 2000; Pigott et al., 1992; Swedo et al., 1989] und Fluoxetin [Ninan et al., 2000] sowie einigen offenen Studien mit Paroxetin [Neudecker et al., 2001] und Fluoxetin [Stanley et al., 1991; Winchel et al., 1992b] wurde die Effektivität der genannten Substanzen gezeigt. Im Gegensatz hierzu konnte in 3 anderen kontrollierten Studien kein signifikanter Behandlungseffekt von Fluoxetin im Vergleich zu Placebo [Christenson et al., 1991b; Streichenwein und Thornby, 1995] oder Warteliste [van Minnen et al., 2003] nachgewiesen werden. Auch in mehreren offenen Studien mit
Fluoxetin [Iancu et al., 1996; Koran et al., 1992], Citalopram [Stein et al., 1997], Fluvoxamin [Christenson et al., 1998; Stanley et al., 1997] und Paroxetin [Minichiello et al., 1994] wurde eine nur geringe Symptomreduktion und/oder niedrige Responderrate von unter 50\% gefunden. In Einzelfällen hat sich bei mangelnder Wirksamkeit von (S)SRI die Zugabe eines Neuroleptikums bewährt [z.B. Ashton, 2001]. Insgesamt ist die Datenlage somit teilweise widersprüchlich und noch unzureichend, die besten Wirkungsnachweise liegen derzeit für Clomipramin vor.

Einige andere Substanzen erwiesen sich in offenen Studien als wirksam: Venlafaxin [Ninan et al., 1998; O'Sullivan et al., 1998], Lithium [Christenson et al., 1991c] und Olanzapin [Stewart und Nejtek, 2003]. Für diese fehlen aber placebokontrollierte Untersuchungen, so dass sie zur Zeit nicht als erste Wahl empfohlen werden können.

Im Langzeitverlauf kam es nach Absetzen einer alleinigen Pharmakotherapie häufig zu Rückfällen, aber auch unter fortgesetzter Medikation nahmen Behandlungserfolge teilweise wieder ab [z.B. Iancu et al., 1996; Ninan et al., 2000]. Daher erscheint die zusätzliche Vermittlung von verhaltenstherapeutischen Strategien in jedem Fall indiziert.

Eine randomisierte Vergleichsstudie von Verhaltenstherapie und Behandlung mit Fluoxetin belegte klar die Überlegenheit ersterer [van Minnen et al., 2003]. Eine eigene offene Studie unter Verwendung von Paroxetin zeigt eine ähnliche Tendenz [Neudecker et al., 2001].

\section{Überblick über die Anwendung von Verhaltenstherapie bei Trichotillomanie}

Die Veröffentlichungen zur verhaltenstherapeutischen Behandlung von Trichotillomanie bestanden über viele Jahre hinweg fast ausschließlich aus Fallbeschreibungen, in denen viele symptombezogene Einzeltechniken wie Gedankenstopp, Selbstbeobachtung, Sammeln der ausgerissenen Haare, Entspannungstraining, Stimuluskontrolle, Selbstinstruktionen, Einsatz aversiver Konsequenzen angewandt wurden [Überblick in Friman et al., 1984]. Da es sich meist um eine Kombination verschiedener Techniken handelte, konnte keine einzelne davon als entscheidend oder besonders hilfreich bei der Trichotillomanie-Behandlung herausgestellt werden.

Dies änderte sich mit der Entwicklung der Technik des «Habit Reversal» [Azrin und Nunn, 1977]. Die Autoren stellten damit eine Methode vor, mit der zunächst Tics relativ erfolgreich behandelt wurden. Bald wurde sie auf andere nervöse Gewohnheiten und Verhaltensweisen wie Nägelkauen, Daumenlutschen und eben auch Trichotillomanie übertragen und spezifiziert. Seitdem ist Habit-Reversal-Training (HRT) die meist angewandte und im verhaltenstherapeutischen Bereich am besten erforschte symptombezogene Methode bei Trichotillomanie. Das Programm ist einzeln und in Gruppen anwendbar. Es besteht aus einer Kombination verschiedener Techniken. Im Mittelpunkt stehen Selbstbeobachtung und das Erlernen alternativer Verhaltensweisen. Ursprünglich hatte es 9 Bestandteile; diese wurden unter anderem von Baer [1994] modifiziert und in 5 Schritten zusammengefasst (Tab. 1).

Baer [1992] begründete die Notwendigkeit systematischer Abgewöhnung mit der Nähe der Trichotillomanie zur Ticstörung. Obwohl diverse Gemeinsamkeiten zur Zwangsstörung bestünden, könne Trichotillomanie nicht mit Exposition und Reaktionsverhinderung behandelt werden, da beim Haare- 
Tab. 1. Bestandteile des HRT [nach Baer, 1994]

\begin{tabular}{ll}
\hline Technik & Beschreibung \\
\hline Aufmerksamkeitstraining & $\begin{array}{l}\text { Selbstbeobachtung (bezogen auf Ort, Zeit, Situation, Gedanken und Gefühle). } \\
\text { Erkennen des genauen motorischen Ablaufs der Handlung. }\end{array}$ \\
Erlernen einer & Finden einer motorischen Gegenbewegung oder -handlung, die das Ausführen des \\
konkurrierenden Reaktion & Verhaltens unmöglich macht. \\
& Einsatz dieser Reaktion bei Auftreten des Impulses. \\
& Beibehalten für mindestens 2 Minuten. \\
& Bezweckt einen generellen inneren Spannungsabbau; soll so die Auftretenshäufigkeit von \\
Entspannungstraining & Impulsen verringern. \\
& Vorzugsweise progressive Muskelrelaxation nach Jacobsen; auch andere Entspannungs- \\
& verfahren sind geeignet. \\
& Individuelle Belohnungen festsetzen. \\
Verstärkung & Bergegenwärtigung der positiven Konsequenzen. \\
& Anwendung des Trainings in allen Situationen, in denen früher Haare ausgerissen wurden. \\
\hline
\end{tabular}

ausreißen wie auch bei Tics, anderen Impulskontrollstörungen und nervösen Gewohnheiten die innere Anspannung mittelfristig eher ansteige, wenn die Handlungsausübung unterbunden wird. Bei genauerer Betrachtung des HRT stellt sich jedoch die Frage, ob es sich nicht dabei sogar um eine fast klassische Form von Exposition handelt. Während der Nichtausübung der Handlung und der motorischen Gegenbewegung kommt es kurzfristig zu einem starken Anstieg des Impulses sowie oft auch assoziierter Gefühle. Außerdem erleben die Patientinnen bei korrekter Ausführung noch während dieser Phase einen spürbaren Rückgang des Impulses und eine völlige Remission nach Ende. Beim HRT wird im Unterschied zur Exposition bei Zwängen deutlich größerer Wert auf die motorische Verhaltensebene gelegt, was bei einer oft automatisierten, schnell verfügbaren und leicht durchzuführenden Handlung wie dem Haareausreißen unbedingt vonnöten ist. Insofern stellt das HRT eine sinnvolle «Mischung» aus Exposition und motorischem Training dar.

Mouton und Stanley [1996] wandten das Habit-Reversal-Konzept im Gruppensetting an. Vier ihrer 5 Patientinnen hatten das Haareausreißen zu Therapieende deutlich reduziert; 2 dieser 4 Personen hatten sich jedoch bis zum 6-Monats-Follow-up wieder verschlechtert. Die Autoren rückten außerdem die zuvor unerwähnte Frage der Behandlungsmotivation stärker in den Fokus der Aufmerksamkeit.

Azrin et al. [1980] führten mit 34 Patientinnen eine randomisierte Untersuchung durch, bei der eine Gruppe mit einer 2stündigen Sitzung zur Implementierung der Habit-ReversalTechnik behandelt wurde, während die andere Gruppe ein so genanntes «Negative Practice Training» erhielt (z.B. geplantes Ausüben der motorischen Anteile der Handlung vor einem Spiegel ohne wirkliches Ausreißen). HRT erwies sich als signifikant überlegen; es wird eine Reduktion des Haareausreißens um $97 \%$ nach 4 Wochen sowie eine gute Stabilität dieses Ergebnisses berichtet. Dies wird jedoch möglicherweise durch einen methodischen Artefakt (mit) verursacht: Die Intensität der Trichotillomanie wurde über detaillierte Selbstbeobachtungsprotokolle erfasst, ergänzt durch regelmäßige telefonische Durchsage der Ergebnisse und Befragung von Angehörigen. Eine so engmaschige Kontrolle kann die Verhaltensraten deutlich senken. Eine Interpretation kann also nur mit großer Vorsicht erfolgen.

In einer offenen Studie untersuchten Lerner et al. [1998] die Wirksamkeit eines kognitiv-behavioralen Programms an 14 erwachsenen Trichotillomanie-Patientinnen. Das Programm bestand aus 9 Einzelsitzungen mit wöchentlichem Abstand. Inhalte waren neben dem üblichen HRT kognitive Umstrukturierung und Rückfallprophylaxe. Die Untersuchung konnte zwar bei 12 Patientinnen einen guten Effekt der Therapie feststellen; dieser hielt jedoch nur bei $31 \%$ der Patientinnen bis zum Follow-up (im Mittel nach 3 Jahren 9 Monaten) an.

HRT hat sich trotz aller Einschränkungen als die Technik der Wahl im Rahmen einer symptomzentrierten verhaltenstherapeutischen Behandlung der Trichotillomanie erwiesen. Allerdings ist durch den Mangel an Katamnesen eine Aussage über den längerfristigen Erfolg dieser Methode derzeit nicht möglich. Weiterhin stellt sich vor dem Hintergrund der zitierten Studien, die prinzipiell von einer hohen Komorbidität von Achse-I- und -II-Störungen ausgehen, die Frage nach dem Sinn der Durchführung allein auf das Haareausreißen abgestimmter (Kurzzeit-)Interventionen. Für rein mikroanalytisch betrachtetes Verhalten mag HRT ausreichen; bei Einbeziehung der Makroebene müsste eine Verhaltenstherapie deutlich komplexer sein. Dazu muss allerdings angemerkt werden, dass alle zitierten Studien aus den USA stammen. Das dortige Gesundheitssystem lässt nur eine sehr geringe Sitzungszahl bei ambulanter Verhaltenstherapie zu, so dass sich die Therapeuten auf das Indexsymptom konzentrieren müssen. Ein letztes Problem besteht darin, dass bis auf wenige Ausnahmen keine Aussagen zur Motivation der Probanden gemacht werden. Da das Ausreißen von Haaren und die darauf folgende (orale) Beschäftigung damit auch einen deutlich positiven An- 
teil hat, dürfte diese eher ambivalent sein. Dabei äußerten Azrin und Nunn bereits in einer ihrer frühen Veröffentlichungen, dass der Motivation der entscheidende Stellenwert zukommt [Azrin und Nunn, 1973].

In Anbetracht der Grenzen einer ausschließlich symptomzentrierten Therapie und der größeren Flexibilität, die hierzulande im Rahmen einer ambulanten Verhaltenstherapie gegeben ist, sprechen die genannten Überlegungen eher für eine individuelle, hypothesengeleitete Form der Therapie. Der Ansatz am Symptom darf dabei nicht unberücksichtigt bleiben. Im stationären Setting ist auch eine Ausgliederung in indikative Gruppen denkbar. Wann und in welchem Rahmen dieser Therapiebaustein jedoch eingefügt wird, bleibt im Einzelfall abzuwägen und hängt außer von der Motivation der Patientinnen hauptsächlich vom Störungsmodell ab. Da diesem zentrale Bedeutung zukommt, soll im folgenden Abschnitt ein Trichotillomanie-spezifisches Störungsmodell anhand eines Fallbeispiels dargestellt werden.

\section{Verhaltenstherapeutisches Störungsmodell und Therapieverlauf am Beispiel einer 31-jährigen Patientin}

Das vorgestellte Modell entstand aus Erfahrungen im Rahmen eines seit 1996 laufenden Trichotillomanie-Forschungsprojektes an der Verhaltenstherapie-Ambulanz und wurde durch Hinweise zur Mikroanalyse des Verhaltens von Mansueto et al. [1997] ergänzt.

Fallskizze: Die verheiratete, kinderlose, zum Zeitpunkt des Erstkontaktes arbeitslose Frau F. hatte aus der Tagespresse von der Möglichkeit erfahren, sich an der Verhaltenstherapie-Ambulanz der Universitätsklinik Hamburg wegen einer Trichotillomanie behandeln zu lassen. Sie riss sich zirka seit ihrem 12. Lebensjahr Haare auf dem Kopf aus, besonders am Scheitel und hinter den Ohren. Das Haar war dort deutlich ausgedünnt und wuchs nur spärlich und unregelmäßig nach. Eine Erklärung für ihr Verhalten hatte sie nicht. Die Intensität schätzte sie auf zirka 300-400 Haare pro Woche. Der Leidensdruck rührte vor allem von ihrer Unzufriedenheit mit ihrem äußeren Erscheinungsbild her. Neben der im Erstgespräch diagnostizierten Trichotillomanie ergaben sich Anhaltspunkte für soziale Ängste und Defizite, ohne dass jedoch die Kriterien für eine soziale Phobie erfüllt waren. Für weitere Symptome oder Störungen, auch im Persönlichkeitsbereich, fanden sich vorerst keine Hinweise. Bisher hatte Frau F. zwei Vorgespräche bei einer tiefenpsychologisch orientierten Therapeutin, sie hatte sich dort aber nicht wohl gefühlt und keine Behandlung begonnen, sowie eine 11-monatige Therapie mit Paroxetin. Letztere führte zwar zu einer Reduktion der Symptomatik um 30\%, wurde von der Patientin aber auf Grund von Nebenwirkungen (v.a. Störung der sexuellen Empfindsamkeit) beendet.

\section{Mikroanalyse der Trichotillomanie}

\section{Aktuelle Auslösebedingungen}

Nur selten beginnt die Verhaltenskette mit dem alleinigen Wunsch, sich Haare auszureißen. Viel häufiger muss eine bestimmte äußere Situation gegeben sein, damit der Impuls entsteht oder seine Auftretenswahrscheinlichkeit erhöht wird.
Dabei spielen auch Konditionierungsprozesse eine Rolle. Beispielsweise kann der Anblick einer Pinzette eine sofortige Assoziation zum Haareausreißen herstellen, so dass die betroffene Person gezielt eine Situation aufsucht, in der die Handlung möglich ist (z.B. Spiegel im Badezimmer). So werden nicht nur Verknüpfungen zu Hilfsmitteln (Pinzette, Spiegel) hergestellt, sondern auch zu speziellen Umgebungen (Schlafzimmer, TV-Sessel, Auto usw.).

Frau F. riss sich überwiegend beim Lesen, Autofahren, Telefonieren und bei subjektiv schwierigen Entscheidungen (z.B. «Was ziehe ich an?») Haare aus. Es fiel ihr anfangs schwer, diese Situationen zu benennen, da die Verbindung von diesen situativen Reizen zum Haareausreißen sehr automatisiert und der bewussten Wahrnehmung kaum mehr zugänglich war. Durch systematische Selbstbeobachtung lernte die Patientin die (äuBeren und internen) Auslöser besser kennen und einordnen.

$\mathrm{Zu}$ den äußeren Bedingungen kommen innere Auslöser. Am weitesten verbreitet sind offenbar (überwiegend negative) emotionale Zustände. Dazu gehören Traurigkeit, Ärger, Frustration usw., aber bei einigen Patientinnen auch freudige Erregung oder Anspannung, z.B. beim Lesen eines Krimis. In der Literatur geht man übereinstimmend davon aus, dass prinzipiell jede Emotion, egal ob positiv oder negativ, vermittelt über einen Spannungsanstieg, den situativen internen Auslöser für das Haareausreißen darstellen kann. Eine zweite Kategorie innerer Auslösereize sind bestimmte sensorische Empfindungen (meist visuell oder taktil). So kann z.B. der Anblick eines andersfarbigen oder das Ertasten eines unregelmäßig dicken Haares den Impuls auslösen, es auszureißen. Als dritte Gruppe interner Auslöser kommen Kognitionen in Frage, wobei diese kaum von den sensorischen Empfindungen zu trennen sind. So kann z.B. der Gedanke «Meine Augenbrauen sind nicht symmetrisch» nur nach einer visuellen Inspektion auftauchen. Symmetriedenken im Sinne von Perfektionsstreben als Auslöser für Haareausreißen kommt allerdings selten vor; wenn doch, scheint eine große Nähe zur Zwangsstörung $\mathrm{zu}$ bestehen.

Zunächst konnte Frau F. innere Auslöser kaum identifizieren. Über Selbstbeobachtungsprotokolle war es ihr jedoch möglich, in manchen Situationen einen unspezifischen Spannungsanstieg oder starke innere Unruhe zu beobachten, was jeweils der Handlung vorausging. Entscheidungssituationen spielten dabei eine wichtige Rolle, wobei sich interne und externe Auslöser mischten. Weiterhin erschien die Patientin in ihrer Alltagsgestaltung «chaotisch». Sie hatte wenig Fähigkeit zur Strukturierung und kam so immer wieder in Stress- und Überforderungssituationen. In diesem Zusammenhang wurde auch ihre fast völlig fehlende Entspannungs- und Genussfähigkeit deutlich. Drittens benannte die Patientin soziale Defizite als Ursache hoher Anspannung, v.a. in den Bereichen Abgrenzung und Vertreten einer Meinung, wobei letzteres überwiegend in engen Beziehungen schwierig war.

Die (innere und äußere) Situation entscheidet allein noch nicht darüber, ob dem Impuls nachgegeben wird oder nicht. Bestimmte diskriminative Reize können sich sowohl fördernd als auch hemmend auf diesen Prozess auswirken. So greift auf $e x$ - 
ternaler Ebene z.B. die Anwesenheit anderer oft zumindest verzögernd in den Prozess ein, während Alleinsein das Ausüben der Handlung fördert. Internal sind einerseits motorische Abläufe geeignet, die Handlung zu fördern (z.B. bestimmte Körperhaltung: aufgestützter Kopf beim Lesen) bzw. zu hemmen (z.B. Beschäftigung der Hände mit anderen Aktivitäten); andererseits können auch kognitive Prozesse eine wichtige Rolle spielen. Viele Patientinnen geben sich die «Erlaubnis» zu reißen, etwa indem sie denken: «Nur dieses eine Haar» oder «Ich habe es mir heute verdient». Andere versuchen, den Prozess mit Gedanken an die negativen Konsequenzen zu unterbinden. Besonders bei ersteren lassen sich unschwer Parallelen zu anderen Zwangsspektrumsstörungen (z.B. Bulimie) wie auch zu süchtigem Verhalten («Nur dieses eine Bier!») erkennen.

Typisch bei Frau F. war das Ausreißen von Haaren, während sie morgens Zeitung las. Sie befand sich in einer gewohnten Körperhaltung (linke Hand blättert in Zeitung, rechte Hand ist aufgestützt, die Finger sind am Kopfhaar). Eine bewusste «Erlaubnis» zum Haareausreißen gab sich die Patientin nie; sie berichtete jedoch, dass es ihr manchmal egal wäre und sie dann einfach reiße.

\section{Verhalten}

Obwohl es auf der motorischen Ebene eine Reihe von Gemeinsamkeiten gibt, variiert das Verhalten von Person zu Person stark. Diese Unterschiede betreffen sowohl die betroffene Stelle (meist Kopf), die Anzahl der Haare (meist einzelne) wie auch das, was nachher mit dem Haar geschieht. Manche Personen werfen es einfach weg, andere beschäftigen sich sehr ausführlich und intensiv damit (Spielen mit den Fingern, Kauen, Zerbeißen, Schlucken, an den Lippen entlang ziehen usw.). Auf physiologischer Ebene wird unterschieden, ob eine Person beim Reißen Schmerz empfindet oder nicht; die meisten tun es nicht. Deswegen besteht auch der Verdacht, es handele sich um einen dissoziationsähnlichen Zustand. Sollte Schmerz auftreten, wird dieser eher im Sinne einer Schreckreaktion zum Beenden der Handlung führen. In Einzelfällen wird ein «bestimmter», als lustvoll erlebter Schmerz intentional gesucht, und das Verhalten kann erst aufhören, wenn dieser erreicht ist. Im Bereich der Emotionen und Kognitionen wird eine Art «Leere» beschrieben; oft werden auch abschweifende Gedankengänge berichtet, die nichts mit dem Haareausreißen zu tun haben.

Frau F. empfand beim Haareausreißen fast nie Schmerz. Sie riss nacheinander einzelne Haare aus und warf diese weg. Manchmal kaute sie vorher auf der Haarwurzel. Sie beschrieb einen Zustand von leichter Trance oder Abwesenheit, «eine Art Käseglocke»; die Realität schien vorübergehend ausgeblendet. Mit ihren Gedanken befand sie sich jeweils «ganz woanders».

\section{Konsequenzen}

Für Patientinnen mit Therapiewunsch sind auf bewusster Ebene zunächst überwiegend die negativen Folgen sichtbar: Ärger auf sich selbst, Scham, das Entstehen kahler Stellen, die Notwendigkeit, diese zu verbergen, in einigen Fällen daraus resultierender sozialer Rückzug, Angst vor intimen Beziehungen usw. Für die Aufrechterhaltung der Störung spielen jedoch eine ganze Reihe verstärkender Konsequenzen eine Rolle: Ablenkung von Stress, Langeweile oder anderen unangenehmen Emotionen; «Belohnung» (v.a. wenn HaareausreiBen mit überwiegend angenehmen Empfindungen verbunden ist); Erfolgserlebnisse (z.B. wenn eine besonders dicke Wurzel gefunden oder ein störendes, unsymmetrisches Haar beseitigt wurde); angenehme sensorische Empfindungen (z.B. Gefühl des Haares auf den Lippen); Selbststimulation; allgemeine Spannungsreduktion auch im Sinne eines Ventils für Aggressionen, Ärger usw. Ungünstigerweise ist es hier wie bei allen anderen neurotischen Störungen auch: Die positiven Konsequenzen treten kurzfristig und damit unmittelbar verhaltenssteuernd auf, während ein Großteil der negativen Folgen erst mittel- bzw. langfristig zur Geltung kommt und in der Situation zunächst wenig Einfluss auf die Handlungsausübung hat. Teilweise kann das sichtbare Entstehen von Kahlheit als Signal an Bezugspersonen gedeutet werden, was in der Regel entweder mit deren Bestrafung oder Forderung nach mehr Zuwendung verbunden ist.

Bereits zum Abschluss der Verhaltensanalyse konnte Frau F. eindeutig eine Reihe von positiven Konsequenzen benennen, z.B. das Abschweifen von belastenden Gedanken, eine als angenehm empfundene innere Leere, eine kurzfristige Spannungsreduktion und den «Käseglockeneffekt». Für das allein durch äußere Situationen getriggerte Reißen ließen sich solche kurzfristigen Verstärker nicht finden. Insgesamt setzten bei allen Situationen Ärger auf sich selbst und Enttäuschung ein; langfristig fühlte sie sich unwohl mit ihrer äußeren Erscheinung. Für eine interaktionelle Funktion der Trichotillomanie fand sich kein Hinweis.

\section{Lebensgeschichtlicher Hintergrund}

Für die Entstehung der Störung fehlt in der Literatur bisher ein klares Modell. Überwiegend wird davon ausgegangen, dass zunächst eine Reihe von externen Stressoren dazu beiträgt, die Trichotillomanie auszulösen. Hierbei werden z.B. genannt: Krankheit oder Tod eines Familienmitgliedes, Umzüge, Trennung von Freunden oder anderen engen Bezugspersonen, Schulbeginn/Schulwechsel/Schulschwierigkeiten, Scheidung der Eltern, Geburt eines Geschwisters oder andere konflikthafte Familienkonstellationen [Christenson und Mansueto, 1999]. Oft geht es dabei nicht um ein einzelnes traumatisierendes Ereignis, sondern eher um einen länger andauernden, innere Spannungen auslösenden Zustand. Ähnlich wie bei anderen psychischen Erkrankungen führt dann ein Mangel an adäquaten Bewältigungsmöglichkeiten zur Manifestation der Symptomatik. Aber auch interne Stressoren wie körperliche Krankheit, Juckreiz oder Menarche können eine Rolle spielen [ebd.]. Diese Stressoren allein erklären jedoch nicht, weshalb es ausgerechnet zum Haareausreißen kommt. Nicht selten erfährt man von Patientinnen auch, dass das Ausreißen von Haaren als Reaktion auf einen Konflikt, meist mit der Mutter, 
stattfand; z.B. wenn das Abschneiden von Haaren als Drohung bzw. Bestrafung eingesetzt und/oder gegen den Willen des Kindes auch durchgesetzt wird [Sticher et al., 1980]. Nach Fallberichten, in denen sexueller Missbrauch eine Rolle spielte, wurde diesem als potenzieller Auslöser eine wichtige Rolle zugeschrieben. Studien zeigten jedoch keine erhöhte Häufigkeit hierfür im Vergleich zur Normalbevölkerung [Christenson et al., 1992].

Darüber hinaus gibt es viele Berichte von Patientinnen über den Beginn ihrer Symptomatik, in denen zunächst keiner der genannten Stressoren zu finden ist. In einer Überblicksarbeit, in die 310 Fälle Eingang gefunden haben, wird von nur 6,8\% eine traumatische Situation oder ein anderer Stressor als auslösend für den Beginn des Haareausreißens angegeben [Graber und Arndt, 1993]. Das Ausreißen von Haaren kann zunächst auch mit einem bewussten Ziel erfolgen: Wegblasen einer Wimper, weil dies Glück bringen soll; Betrachten eines Haares unter dem Mikroskop im Biologieunterricht; Entfernen von störenden grauen Haaren usw. Auch Modelllernen, also die Beobachtung des Verhaltens an einer anderen Person, kann den Anstoß geben, Haare auszureißen. Möglicherweise spielt auch genetische Veranlagung eine Rolle. So hat eine Untersuchung von Lenane et al. [1992] eine tendenziell höhere Lebenszeitprävalenz von Zwangsstörungen bei Verwandten ersten Grades ergeben. Inwieweit diese Veranlagung zu zwanghaften Gewohnheiten tatsächlich neurobiologisch nachweisbar ist, muss sich noch herausstellen. Ebenso schwierig ist ein Zusammenhang zwischen dem Hormonhaushalt und Trichotillomanie herzustellen. Der Erstauftrittsgipfel um die Pubertät herum legt diese These nahe; auch die oft berichtete Zunahme des Verhaltens vor Einsetzen der Menstruation [Keuthen et al., 1997] spricht dafür. Es lässt sich jedoch schwer unterscheiden, ob dies rein biologisch bedingt oder über emotionale und kognitive Prozesse vermittelt ist.

Generell ist es schwierig, aus so unterschiedlichen Angaben und Beobachtungen auf gemeinsame Faktoren bei der Entstehung der Trichotillomanie zu schließen. Am ehesten kommt ein multikonditionales Modell in Frage, das von bestimmten auslösenden Bedingungen ausgeht, die den Boden für die Entwicklung eines solchen Verhaltens schaffen. Der Ausbruch der Symptomatik kann dann durch zusätzliche Stressoren und/oder Zufall geschehen. Aus lerntheoretischer Sicht interessiert an diesem Punkt der Pathogenese vor allem die Verstärkung, die über das Aufgeben oder Fortführen der «neuen» Verhaltensweise entscheidet. Bei Patientinnen mit Trichotillomanie erfolgt vermutlich sowohl positive als auch negative Verstärkung, was zu einer schnellen Generalisierung und Chronifizierung des Verhaltens führt.

Frau F. beschrieb ihre Kindheit als glücklich und unkompliziert. Sie hatte jedoch kaum Möglichkeiten, von sich aus Ideen zu entwickeln. Die Eltern waren dominante Respektspersonen; was sie sagten, wurde gemacht. Dies führte lerngeschichtlich bereits relativ früh zu einer passiven Grundhaltung. Nach einem Umzug war die Notwendigkeit zur Anpassung im Elternhaus mangels Alternativen im sozialen Bereich noch größer. Durch den einseitigen Kommunikationsablauf in der Familie sowie den eingeschränkten Umgang mit anderen Kindern wurde der Grundstein für die Entwicklung deutlicher sozialer Defizite gelegt.

In den ersten Schuljahren bekam Frau F. von ihren Eltern nur wenig Unterstützung. Leistungsschwierigkeiten wurden nicht ernst genommen, sie fühlte sich allein gelassen. Auf der Grundlage ihrer bereits bestehenden Passivität entwickelte sie eine gewisse Reaktanz gegenüber Anforderungen. Motorisch war sie eher unruhig und aufgedreht; offenbar fehlten ihr ausreichende Kompensationsmöglichkeiten.

Der Beginn der Symptomatik fiel in einen Zeitraum, in welchem sich Frau F. auf Grund hoher Leistungserwartungen am Gymnasium überfordert und gestresst sah. Sie hatte große Ängste und Defizite bezüglich mündlicher Mitarbeit. Eine Entlastung im Elternhaus fand nicht statt. Die Patientin hatte sich auch nicht darum bemüht, was den Eindruck vom bereits vorher wenig hilfreichen Umgang mit Problemen und Schwierigkeiten bestätigt. Das Haareausreißen begann als Mittel der kurzfristigen Spannungsreduktion in Überforderungssituationen, aber auch als Ablenkung. Es generalisierte auf Grund dieser kurzfristig äußerst angenehmen Konsequenzen sehr schnell auf Situationen, in denen eine Beschäftigung bzw. Ablenkung einen unangenehmen Zustand beenden konnte (negative Verstärkung). Das fast reflexartige Einsetzen der Trichotillomanie in allen auch nur ansatzweise spannungsauslösenden Situationen begünstigte eine Automatisierung dieses Verhaltens als «Standardmethode» im Sinne einer teilweise unbewusst ablaufenden Angewohnheit.

Als wichtige aufrechterhaltende Bedingung wurde hohe innere Anspannung bei fehlender Toleranz für Leerlaufsituationen deutlich. Dies ging einher mit einer mangelnden Wahrnehmungsfähigkeit der eigenen Gefühle sowie fast völlig fehlender Entspannungs- und Genussfähigkeit.

\section{Testdiagnostik}

Prinzipiell ist es im Sinne von Patientinnen und Therapeuten, die Veränderungen in einer Verhaltenstherapie auch testdiagnostisch zu begleiten. Beim Versuch, die jeweils aktuelle Intensität der Trichotillomanie möglichst objektiv zu erfassen, stößt man jedoch auf Schwierigkeiten, die in der Natur des Symptoms liegen. Zunächst einmal ist das Verhalten oft unbewusst, so dass Patientinnen nur schwer Angaben zur Dauer des täglichen Ausreißens oder zur Anzahl der Haare machen können. Dem wäre zwar durch Selbstbeobachtung oder Sammeln der Haare zu begegnen; dies wiederum beeinflusst das Verhalten sehr. In der Hamburger Studie geschah es beispielsweise, dass einige Patientinnen die Trichotillomanie gänzlich stoppten, weil sie das Führen von Protokollen so unangenehm fanden. Leider war dieser Effekt nie von Dauer. Ein drittes Problem liegt in der Veränderungsmessung. Die meisten Patientinnen verbessern während einer Verhaltenstherapie ihre Wahrnehmungsfähigkeit für das Haareausreißen. Ein höherer Messwert zu Therapieende muss deshalb nicht für eine $\mathrm{Zu}-$ nahme der Störungsintensität stehen. Valide Selbst- und Fremdratings in deutscher Sprache existieren nicht. Aus dem englischsprachigen Raum sind die Psychiatric Institute Trichotillomania Scale PITS [Winchel et al., 1992a] oder die Massachusetts General Hospital Hairpulling Scale MGH-HS [Keuthen et al., 1995] bekannt. Eine Fremdeinschätzung durch Beobachtung, Messung usw. ist vor allem zur Verlaufsbeurteilung schwierig, da die Haare oft nur extrem langsam und spärlich 
nachwachsen. Ein holländisches Forscherteam kombinierte in seiner Studie Selbstratings mit Videoaufzeichnungen, um eine möglichst optimale Einschätzung zu erhalten [Van Minnen et al., 2003]. Dieser Aufwand ist in der alltäglichen Praxis jedoch unrealistisch.

Frau F. wurde mit der ins Deutsche übersetzten PITS untersucht. Sie erreichte vor Beginn der Therapie 14 von 42 möglichen Punkten. Zusätzlich wurden zur testdiagnostischen Abklärung der Komorbidität das Beck Depressionsinventar (BDI), das Hamburger Zwangsinventar (Kurzform; HZI-K), der Unsicherheits-Fragebogen (U-Fragebogen) und das Inventar klinischer Persönlichkeitsakzentuierungen [IKP; Andresen, 2004] eingesetzt. Die Patientin war in den drei erstgenannten Verfahren unauffällig, was sich mit der klinischen Eingangsdiagnostik deckt. Im Persönlichkeitsinventar ergaben sich Hinweise auf passive, ängstlich-vermeidende und asthenische Züge, auch hier in Übereinstimmung mit der klinischen Diagnose ohne das Vorliegen einer Persönlichkeitsstörung.

\section{Therapieverlauf und -ergebnisse}

Der Therapieverlauf orientierte sich an den strategischen Modellvorgaben von Hand [2002], bei denen neben der Mikroanalyse zum Symptom vor allem den Entstehungs- und aufrechterhaltenden Bedingungen und Funktionen entscheidender Stellenwert zukommt. Die Interventionen werden dabei im Gegensatz zu den oben genannten Studien nicht diagnosenspezifisch, sondern anhand der in der Verhaltensanalyse gewonnenen Hypothesen ausgewählt.

Die Patientin nannte nach Abschluss der Verhaltensanalyse folgende Therapieziele:

1. Reduktion des Haareausreißens

2. Reduktion der inneren Anspannung, mehr Gelassenheit

3. Verbesserung der sozialen Kompetenz (Meinung vertreten, Neinsagen können)

Diese Ziele stimmten weitgehend mit denen der Therapeutin überein, die Ziel Nr. 1 jedoch eher als Folge des Erreichens der Ziele 2 und 3 sah und als weiteres Ziel eine Reduktion der Defizite in Gefühlswahrnehmung und -ausdruck hinzufügte. In einer ausführlichen Besprechung der Hypothesen wurde deutlich, dass die Patientin zu diesem Zeitpunkt eigentlich keinen Zugang zum Zusammenhang zwischen ihrem Umgang mit Emotionen und dem Symptom hatte und demnach nicht motiviert war, zu Beginn der Therapie an diesen Themen zu arbeiten. Wir vereinbarten daher, mit dem HRT zu beginnen. Implizites Therapeutenziel war es, der Patientin auf diesem Weg die aufrechterhaltenden Bedingungen zu verdeutlichen.

Frau F. war zunächst begeistert von dieser Methode und hatte in vielen Situationen auch Erfolg. Sie beobachtete ein Ansteigen der inneren Anspannung, welche aber nach etwa 1 Minute einen Gipfel erreichte und dann wieder abfiel, ohne dass sie ein Haar ausgerissen hatte. Im Nachhinein betrachtet waren dies genau jene Situationen, in denen tatsächlich die Gewohnheit die entscheidende Rolle gespielt hatte. In einigen Situationen, z.B. wenn der innere Stress zu groß war, funktionierte das HRT jedoch nicht. Die Patientin hielt entweder nicht durch oder riss danach trotzdem Haare aus. In anderen Situationen setzte die Patientin das HRT nicht ein und riss, ohne Widerstand überhaupt in Erwägung gezogen zu haben.

An diesem Verlauf zeigt sich das Risiko einer nur vorübergehenden Besserung bei einem zu stark symptomorientierten Vorgehen. Obwohl das HRT von der Patientin erlernt, geübt und erprobt wurde und kurzfristig auch zu gutem Erfolg ge- führt hatte, zeigten sich im weiteren Verlauf massive Schwierigkeiten bei der konsequenten Umsetzung. Ähnliche Befunde sind aus der Untersuchung von Stanley und Mouton [1996] bekannt. Die Autoren berichten nach anfänglicher guter Response auf ein im Gruppensetting durchgeführtes HRT über Noncompliance bei den häuslichen Übungen, unregelmäßige Teilnahme, fehlende Bereitschaft für ein Gruppenkonzept sowie Festhalten an einem biologischen Genesemodell. Eine andere Studie (kognitiv-behaviorale Therapie) mit Follow-up [Lerner et al., 1998], die im Einzelsetting durchgeführt wurde, hatte eine hohe Dropout-Rate, was ebenfalls für eine unklare Behandlungsmotivation spricht. Weiterhin berichten Keuthen et al. [2001] aus einer retrospektiven Untersuchung von Trichotillomanie-Patientinnen, die sowohl Verhaltens- als auch pharmakologische Therapien erhalten hatten, dass initial zwar ein guter Therapieerfolg nachweisbar war, dieser jedoch ein Plateau erreichte bzw. wieder abfiel, obwohl sich ein Teil der Patientinnen noch in weiterführender Behandlung befand. Es wird hier ein Zusammenhang zu einem niedrigen Selbstwertgefühl hergestellt, der nach Ansicht der Autoren allerdings sekundär zur Trichotillomanie besteht.

Gerade bei Impulskontrollstörungen, bei denen das Problemverhalten ja auch einen als ausgesprochen positiv erlebten Anteil hat, muss von starker Ambivalenz bezüglich einer Symptomreduktion bei einem Teil der Patienten ausgegangen werden. Dies entspricht auch unseren klinischen Erfahrungen. Umso wichtiger erscheint daher eine individuelle, hypothesengeleitete Behandlungsplanung, bei der auch beurteilt wird, ob 1) andere Problembereiche (wie z.B. ein niedriges Selbstwertgefühl oder soziale Defizite) auch primär, also (mit-)auslösend für die Symptomatik sein können und ob 2) die Veränderungsmotivation gezielt gefördert werden sollte.

Mit dem ersten Problem (HRT war zu schwierig) wurden die Ziele Nr. 2 und 3 der Patientin aktualisiert. Anhand der Selbstbeobachtungsprotokolle wurden zum einen Entscheidungssituationen als Auslöser für hohe innere Anspannung identifiziert; zum anderen lösten Konflikte, in denen es der Patientin nicht gelang, sich abzugrenzen und/oder für ihre Bedürfnisse zu sorgen, Stress aus. Die Entscheidungssituationen betrafen meist banale Dinge (z.B. «Was ziehe ich an?») und wurden sowohl kognitiv als auch mit kleineren Übungen (vorgegebene Zeit bis zur Entscheidung, bewusst falsche Entscheidungen treffen usw.) bearbeitet. Bezüglich der sozialen Defizite wurde nach einer Klärung des gewünschten Zielverhaltens mit Rollenspielen mit Videofeedback sowie häuslichen Übungsaufgaben gearbeitet. Deutlich wurde dabei der Wunsch der Patientin, niemanden zu kränken und sich lieber anzupassen, was bisher zu einem Aggressionsstau geführt hatte, der unter anderem durch das Haareausreißen abreagiert wurde.

In beiden Bereichen machte die Patientin gute Fortschritte und zeigte sich hoch motiviert, da ihr nun der Zusammenhang zur Hauptsymptomatik viel klarer war. Dennoch stagnierte die Trichotillomanie weiter. In der Therapie stellte sich mit dem zweiten Problem (Nichtanwendung des HRT) die Frage, ob Frau F. tatsächlich gewillt war, völlig auf das Haareausreißen zu verzichten. Aussagen wie «Dann habe ich mir ein Haar gegönnt» legten diese Frage nahe. Die Patientin schien Stress, schwierige Lebensumstände usw. als «Alibi» für erneutes Haareausreißen zu «missbrauchen». In Anlehnung an kognitive Bestandteile der Behandlung von Abhängigkeitsstörungen [z.B. Beck et al., 1995] konnte herausgearbeitet 
werden, dass die Patientin sich das Haareausreißen «erlaubte» und auf die erlernten Alternativstrategien verzichtete. In einem «Abschiedsbrief an das Haareausreißen» bestätigte Frau F., dass dieses über Jahrzehnte hinweg ein vertrauter Begleiter gewesen sei, der durchaus auch seine guten Seiten hatte und habe. Wir ergänzten den Brief mit der Frage, wie wohl ein Leben nach dem Haareausreißen aussähe. Die Patientin reagierte sehr gut auf entsprechende Imaginationsübungen. Sowohl beim Vorlesen dieses Briefes als auch während der Vorstellungsübungen kamen massive Gefühle von Trauer und Verzweiflung hoch, nachdem sich die Patientin zu einem wirklichen «Verzicht» auf ihre Gewohnheit entschlossen hatte. Dies wurde für das Erlernen eines veränderten Umgangs mit unangenehmen Emotionen genutzt, wobei wir unter anderem mit Ideen aus dem hierfür sehr hilfreichen Buch von Görlitz [2001] arbeiteten. Im Anschluss fand eine erneute Zielklärung statt; seit diesem Zeitpunkt riss die Patientin kaum noch Haare aus.

Obwohl im beschriebenen Therapieabschnitt auch Interventionen zur Verbesserung der sozialen und emotionalen Kompetenz durchgeführt wurden, schien die Bearbeitung der Ambivalenz hinsichtlich des Aufgebens der Trichotillomanie zentral zu sein. Dieser Prozess schaffte vor allem für die Patientin deutlich mehr Verständnis hinsichtlich ihrer bisherigen Schwierigkeiten, das Symptomverhalten abzubauen. Diese waren nun nicht mehr unerklärlich oder gar «böswillig», sondern nachvollziehbar [vgl. Rufer, 2003]. Leider existieren unseres Wissens keine Studien zur Trichotillomanie oder anderen Störungen der Impulskontrolle, wie sie für einige der stoffgebundenen Suchtstörungen durchgeführt wurden [z.B. Kröger et al., 2004]), die das Zusammenspiel von SymptomInterventionen und Motivation zum Gegenstand haben.

In den verbleibenden Sitzungen, die nun in größeren Abständen stattfanden, ging es neben der Reflexion der Therapie vorwiegend um Rückfallprophylaxe. Die Therapie wurde nach 21 Sitzungen in beiderseitigem Einvernehmen beendet. Die Patientin war zu diesem Zeitpunkt in der 8 . Woche schwanger und hatte für sich die Überzeugung gewonnen, dass das lang ersehnte Eintreten der Schwangerschaft auch mit dem emotionalen Wandel während der Therapie zu tun hatte. Allerdings führte dieser $\mathrm{Zu}$ stand sowohl emotional als auch physiologisch zu vermehrtem Stress, was zu einer nochmaligen Zunahme der Trichotillomanie führte. Frau F. zeigte sich jedoch sicher, dies allein wieder in den Griff zu bekommen.

Die Werte der Testdiagnostik präsentierten sich wie folgt: PITS 11 Punkte (Rückgang um zirka 21\%); Angaben der Patientin: 20-40 Haare pro Woche; Nachuntersuchungstermin nach 3 Monaten: PITS 8 Punkte; U-Fragebogen, HZI-K und BDI weiterhin unauffällig; IKP: in allen Skalen unauffällig.

\section{Zusammenfassung und Diskussion}

Im Fallbeispiel wurde eine hypothesengeleitete, multimodale Verhaltenstherapie vorgestellt, bei der sowohl Störungsmodell als auch Art und Reihenfolge der Interventionen transparent gehalten und von der Patientin mitbestimmt wurden. Dieses «join the client where he/she is» führte neben einer guten therapeutischen Beziehung zur Wahl des HRT als erster Hauptintervention. Eine differenzierte Durchführung dieses Trainings konnte einen Teil des Problemverhaltens bereits eliminieren. Andererseits ergaben sich die entscheidenden Hin- weise auf die aufrechterhaltenden Bedingungen der Symptomatik, zu denen die Patientin vorher keinen direkten Zugang herstellen konnte und die in der Folge in den Behandlungsfokus rückten. Erst durch die Erhöhung von sozialer und emotionaler Kompetenz und Entspannungsfähigkeit sowie durch gezielte Motivationsförderung konnte ein befriedigendes Therapieresultat erzielt werden. Etwas überraschend war, dass die anfangs erfassten und als relativ zeitstabil geltenden Persönlichkeitsakzentuierungen (passiv, ängstlich-vermeidend und asthenisch) am Ende dieser doch relativ kurzen Therapie keine Rolle mehr spielten. Da es sich nicht um Bestandteile einer ausgeprägten Persönlichkeitsstörung handelte, waren diese offenbar durch die genannten Interventionen im Bereich der aufrechterhaltenden Bedingungen indirekt gut beeinflussbar.

Trotzdem ist die prognostische Einschätzung mit Skepsis zu betrachten. Obwohl die Patientin ihren PITS-Wert bis zum ersten Follow-up weiter reduzieren konnte, wird die zukünftige Intensität des Symptoms Schwankungen unterliegen, die bei mangelnder Aufmerksamkeit schnell wieder zu einer Zunahme der Trichotillomanie führen können. Außerdem ist es schwer, die benötigte hohe Motivation langfristig aufrechtzuerhalten, da sich ein sichtbarer Erfolg nur langsam einstellen wird.

Verglichen mit den bisherigen Darstellungen zur Verhaltenstherapie bei Trichotillomanie zeigt dieses Beispiel, dass eine alleinige Symptomtherapie ohne Berücksichtigung der komorbiden Psychopathologie kaum ausreichen kann. Kurzfristig mag sie zwar eine schnelle Besserung bewirken; mittelund langfristig spielen jedoch Komorbidität und Motivation die entscheidende Rolle. Die Motivation stellt ab einem bestimmten Punkt in der Therapie aus unserer Sicht das Hauptproblem dar. Während die verhaltenstherapeutischen Standardinterventionen in entsprechender Auswahl und Abstimmung gut geeignet sein können, den negativ verstärkten Anteil der Symptomatik zu bearbeiten und Alternativverhaltensweisen zu erlernen, dürfte es für die meisten Patientinnen sehr schwer sein, sich dauerhaft die positiven Aspekte der Trichotillomanie zu versagen. Dies wird besonders dann schwierig, wenn das nachgewachsene Haar in gewisser Weise das Ausreißen erlaubt, ohne dass dies gleich sichtbar werden muss. In den geschilderten standardisierten Gruppentherapien findet dies alles in keiner Weise Beachtung. Aber auch im Einzelsetting ist das Patentrezept zum Umgang damit noch nicht gefunden. In Einzelfällen mögen Ziel- und Belohnungsszenarien oder Interventionen wie der «Abschiedsbrief vom Symptom» helfen - eventuell auch der fortgesetzte Besuch von Selbsthilfegruppen. Insgesamt jedoch sollten Optimismus und gesunder Realismus bei Betroffenen und Therapeuten ausgewogen sein; ist der Leidensdruck im Sinne der nach außen sichtbaren Einschränkung erst einmal verschwunden, hat die Verhaltenstherapie möglicherweise ihre Grenze erreicht, obwohl noch immer Haare ausgerissen werden. 


\section{Literatur}

Andresen B: Inventar Klinischer Persönlichkeitsakzentuierungen IKP. Göttingen, Hogrefe, 2004.

Ashton AK: Olanzapine augmentation for trichotillomania. Am J Psychiatry 2001;158:1929-1930.

Azrin NH, Nunn RG: Habit-reversal: A method of eliminating nervous habits and tics. Behav Res Ther 1973;11:619-628

Azrin NH, Nunn RG: Habit Control in a Day. New York, Simon und Schuster, 1977.

Azrin NH, Nunn RG, Frantz SE: Treatment of hairpulling (trichotillomania): A comparative study of habit reversal and negative practice training. J Behav Ther Exp Psychiatry 1980;11:13-20.

Baer L: Behavior therapy for obsessive-compulsive disorder and trichotillomania. Implications for Tourette syndrome. Adv Neurol 1992;58:333-340.

Baer L: Alles unter Kontrolle. Bern, Huber, 1994.

Beck AT, Wright F, Newman C, Liese B: Kognitive Therapie der Sucht. Weinheim, Psychologie Verlags Union, 1995

Christenson GA: Trichotillomania - from prevalence to comorbidity. Psychiatric Times 1995;12:44-48.

Christenson GA, Mansueto CS: Trichotillomania: Descriptive characteristics and phenomenology; in Hollander E (ed): Trichotillomania. Washington, DC, American Psychiatric Press, 1999, pp 1-41.

Christenson GA, Mackenzie TB, Mitchell JE: Characteristics of 60 adult chronic hair pullers. Am J Psychiatry 1991a;148:365-370.

Christenson GA, Mackenzie TB, Mitchell JE, Callies BA: A placebo-controlled, double-blind crossove study of fluoxetine in trichotillomania. Am J Psychiatry 1991b;148:1566-1571.

Christenson GA, Popkin MK, Mackenzie TB, Realmuto GM: Lithium treatment of chronic hair pulling. J Clin Psychiatry 1991c;52:116-120.

Christenson GA, Pyle RL, Mitchell JE: Estimated lifetime prevalence of trichotillomania in college students. J Clin Psychiatry 1991d:52:415-417.

Christenson GA, Chernoff Clementz E, Clementz BA: Personality and clinical characteristics in patients with trichotillomania. J Clin Psychiatry 1992;53:407-413.

Christenson GA, MacKenzie TB, Mitchell JE: Adul men and women with trichotillomania. A comparison of male and female characteristics. Psychosomatics 1994;35:142-149.

Christenson GA, Crow SJ, Mitchell JE, Mackenzie TB, Crosby RD, Falls J: Fluvoxamine in the treatment of trichotillomania: A 8-week, open-label study. CNS Spectrums 1998;3:64-71

Friman PC, Finney JW, Christophersen ER: Behavioral treatment of trichotillomania: An evaluative review. Behav Ther 1984;15:249-265.

Görlitz G: Körper und Gefühl in der Psychotherapie. Stuttgart, Klett-Cotta, 2001

Graber J, Arndt WB: Trichotillomania. Compr Psychiatry 1993:34:340-346

Hand I: Multimodale Verhaltenstherapie; in Schneider W (Hrsg): Lehrbuch der Psychotherapie und Psychosomatischen Medizin. Stuttgart, Schattauer, 2002, pp $560-568$.
Hollander E, Wong CM: Obsessive-compulsive spectrum disorders. J Clin Psychiatry 1995;56:43-46. Iancu I, Weizmann A, Kindler S, Sasson Y, Zohar J: Serotonergic drugs in trichotillomania: Treatment results in 12 patients. J Nerv Ment Dis 1996;184:641-644. Keuthen NJ, O'Sullivan RL, Ricciardi JN, Shera D, Savage CR, Borgmann AS, Jenike MA, Baer L: The Massachusetts General Hospital (MGH) Hairpulling Scale: 1. Development and factor analyses. Psychother Psychosom 1995;64:141-145.

Keuthen NJ, O'Sullivan RL, Hayday CF, Peets KE, Jenike MA, Baer L: The relationship of menstrual cycle and pregnancy to compulsive hairpulling. Psychother Psychosom 1997;66:33-37.

Keuthen NJ, Fraim C, Deckersbach T, Dougherty DD, Baer L, Jenike MA: Longitudinal follow-up of naturalistic treatment outcome in patients with trichotillomania. J Clin Psychiatry 2001;62:101-107.

Koran LM, Ringolg A, Hewlett W: Fluoxetine for trichotillomania: An open clinical trail. Psychopharmacol Bull 1992;28:145-149.

Kröger C, Metz K, Bühler A: Tabakentwöhnung bei Patienten in Rehabilitationskliniken. Suchtmed 2004;6: 61-66.

Lenane MC, Swedo SE, Rapoport JL, Leonard H, Sceery W, Guroff JJ: Rates of obsessive compulsive disorder in first degree relatives of patients with trichotillomania: A research note. J Child Psychol Psychiatry 1992;33:925-933.

Lerner J, Franklin ME, Meadows EA, Hembree E, Foa EB: Effectiveness of a cognitive-behavioral treat ment program for trichotillomania: An uncontrolled evaluation. Behav Ther 1998;29:157-171.

Mansueto CS, Stemberger RM, Thomas AM, Golomb RG: Trichotillomania: A comprehensive behavioral model. Clin Psychol Rev 1997;17:567-577.

Minichiello WE, O'Sullivan RL, Osgood-Hynes D, Baer L: Trichotillomania: Clinical aspects and treatment strategies. Harv Rev Psychiatry 1994;1:336-344.

Mouton SG, Stanley MA: Habit reversal training of trichotillomania: A group approach. Cog Behav Pract 1996;3:159-182.

Neudecker A, Rufer M, Hand I, Zaninelli R: Paroxetin versus multimodale Verhaltenstherapie bei Trichotillomanie: Ergebnisse einer Pilotstudie. Nervenheilkunde 2001;20:148-149.

Ninan PT, Knight RN, Kirk L, Rothbaum BO, Kelsey J, Nemeroff CB: A controlled trial of venlafaxine in trichotillomania: Interim phase I results. Psychopharmacol Bull 1998;34:221-225.

Ninan PT, Rothbaum BO, Marsteller FA, Knight RN, Eccard MB: A placebo-controlled trial of cognitive-behavioral therapy and clomipramine in trichotillomania J Clin Psychiatry 2000;61:47-50.

O'Sullivan RL, Keuthen NJ, Rodriguez D, Goodchild P, Christenson GA, Rauch SL, Jenike MA, Baer L: Venlafaxine treatment of trichotillomania: An open series of ten cases. CNS Spectrums 1998;3:56-63.
Pigott TA, L'Heueux F, Grady TA, Bernstein S, Dubbert B, Rubinstein C, Hill J, Murphy D: Controlled comparison of clomipramine and fluoxetine in trichotillomania. Presented at 31st Annual Meeting of the American College of Neuropsychopharmacology, San Juan, Puerto Rico, 1992

Rufer M: Wenn sich während psychotherapeutischen Behandlungen nichts verändert. Psychotherapeut 2003;48:264-266

Schlosser S, Black DW, Blum N, Goldstein RB: The demography, phenomenology, and family history of 22 persons with compulsive hair pulling. Ann Clin Psychiatry 1994:6:147-152.

Stanley MA, Mouton SG; Trichotillomania treatmen manual; in Van Hasselt VB, Hersen M (eds): Sourcebook of Psychological Treatment Manuals for Adul Disorders: New York, Plenum Press, 1996, pp 657-687. Stanley MA, Bowers TC, Swann AC, Taylor DJ: Treatment of trichotillomania with fluoxetine. J Clin Psychiatry 1991;52:282

Stanley MA, Swann AC, Bowers TC, Davis ML, Taylor DJ: A comparison of clinical features in trichotillomania and obsessive-compulsive disorder. Behav Res Ther 1992;30:39-44.

Stanley MA, Breckenridge JK, Swann AC, Freeman EB: Fluoxamine treatment of tichtillomania. J Clin Psychopharmacol 1997;17:278-283.

Stein DJ, Bouwer C, Maud CM: Use of selective serotonin reuptake inhibitor citalopram in treatment of trichotillomania. Eur Arch Psychiatry Clin Neurosci 1997;247:234-236.

Stewart RS, Nejtek VA: An open-label flexible-dose study of olanzapine in the treatment of trichotillomania. J Clin Psychiatry 2003;64:49-52.

Sticher M, Abramovits W, Newcomer VD: Trichotillomania in adults. Cutis 1980;26:90, 97-101.

Streichenwein SM, Thornby JI: A long-term, doubleblind, placebo-controlled crossover trial of the efficacy of fluoxetine for trichotillomania. Am J Psychiatry 1995;152:1192-1196.

Swedo SE, Leonard HL, Rapoport JL, Lenane MC, Goldberger EL, Cheslow DL: A double-blind comparison of clomipramine and desipramine in the treatment of trichotillomania (hair pulling). N Engl J Med 1989; 321:497-501.

Swedo SE, Leonard HL: Trichotillomania. An obsessive compulsive spectrum disorder? Psychiatr Clin North Am 1992;15:777-790.

Van Minnen A, Hoogduin K, Keijsers G, Hellenbrand I, Hendriks G: Treatment of trichotillomania with behavioral therapy or fluoxetine. Arch Gen Psychiatry 2003;60:517-522.

Winchel RM, Jones JS, Molcho A, Parsons B, Stanley B, Stanley M: The Psychiatric Institute Trichotillomania Scale (PITS). Psychopharmacol Bull 1992a;28: 463-476.

Winchel RM, Jones JS, Stanley B, Molcho A, Stanley M: Clinical characteristics of trichotillomania and its response to fluoxetine. J Clin Psychiatry 1992b;53: 304-308 